M. Schlosser · J. P. Banga • A. M. Madec $\cdot$ K. A. Binder $\cdot$

M. Strebelow $\cdot$ I. Rjasanowski $\cdot$ R. Wassmuth $\cdot$

L. K. Gilliam • D. Luo - C. S. Hampe

\title{
Dynamic changes of GAD65 autoantibody epitope specificities in individuals at risk of developing type 1 diabetes
}

Received: 11 October 2004 / Accepted: 21 December 2004 / Published online: 16 April 2005

(C) Springer-Verlag 2005

\begin{abstract}
Aims/hypothesis: Progression to type 1 diabetes is associated with intramolecular epitope spreading to disease-specific antibody epitopes located in the middle region of glutamic acid decarboxylase 65 (GAD65). Methods: The relationship between intramolecular epitope spreading of autoantibodies specific to GAD65 in relation to the risk of developing type 1 diabetes was tested in 22 high-risk individuals and 38 low-risk individuals. We determined the conformational epitopes in this longitudinal study by means of competition experiments using recombinant $\mathrm{Fab}$ of four GAD65-specific monoclonal antibodies. Results: Sera from high-risk children in the preclinical stage recognise a spe-
\end{abstract}

M. Schlosser

Institute of Pathophysiology,

Ernst Moritz Arndt University of Greifswald,

Karlsburg, Germany

J. P. Banga

Division of Medicine, GKT School of Medicine,

London, UK

A. M. Madec

INSERM, Laennec Faculty of Medicine,

University of Lyon,

Lyon, France

K. A. Binder · L. K. Gilliam · D. Luo · C. S. Hampe $(\bowtie)$

Department of Medicine, University of Washington,

P.O. Box 357710 Seattle, WA, 98195, USA

e-mail: champe@u.washington.edu

Tel.: +1-206-5435284

Fax: +1-206-5433169

M. Strebelow

L-A-D GmbH Hennigsdorf,

Branch Karlsburg,

Germany

I. Rjasanowski

Center of Diabetes and Metabolic Disorders,

Karlsburg, Germany

R. Wassmuth

University of Düsseldorf,

Düsseldorf, Germany cific combination of GAD65 antibody epitopes located in the middle and the C-terminus of GAD65. High risk of progressing to disease is associated with the emergence of antibodies specific for conformational epitopes at the Nterminus and the middle region. Binding to already established antibody epitopes located in the middle and at the $\mathrm{N}$-terminus increases and shows a significant relation $(p=$ 0.005 ) with HLA, which confers risk of developing diabetes. Conclusions/interpretation: In type 1 diabetes, GAD65 antibodies are initially generated against the middle and C-terminal regions of GAD65. In genetically predisposed subjects the autoimmune response may then undergo intramolecular epitope spreading towards epitopes on the $\mathrm{N}$-terminus and further epitopes located in the middle. These findings clearly demonstrate that the GAD65 autoantibody response in the preclinical stage of type 1 diabetes is dynamic and related to the HLA genotypes that confer risk of diabetes. GAD65-specific Fab should prove useful in predicting progression from islet autoimmunity to clinical onset of type 1 diabetes.

Keywords Autoantibody $\cdot$ Epitope $\cdot$ Type 1 diabetes

Abbreviations GAD65: 65-kDa isoform of glutamate decarboxylase - GAD65Ab: GAD65 autoantibody - IA-2: islet antigen-2 $\cdot$ PGS: Protein G sepharose $\cdot \mathrm{rFab}$ : recombinant Fab

\section{Introduction}

Autoantibodies to islet cell antigens are present in the majority of patients at the clinical diagnosis of type 1 diabetes. These autoantibodies are primarily directed against three defined antigens: the $65-\mathrm{kDa}$ isoform of glutamate decarboxylase (GAD65), insulin and the protein tyrosine-like islet antigen-2 (IA-2). All three autoantibodies can also be found in the serum of pre-diabetic individuals, sometimes years before clinical onset of the disease [1-4]. Their combined presence makes it possible to identify individuals at high risk of developing type 1 diabetes [5-9]. While auto- 
antibodies to GAD65, IA-2 and insulin are clearly markers for type 1 diabetes, up until recently they were viewed as the product of a bystander response to the autoimmune destruction of the pancreatic beta cells. Recent findings suggest that autoantibodies to GAD65 (GAD65Ab) may play a pathogenic role by modulating the GAD65 presentation to the immune system [10-12].

Epitopes recognised by GAD65Ab at the clinical onset of diabetes have been the focus of many studies [13-16]. We and others have established significant differences in the GAD65Ab epitope patterns present in patients with type 1 diabetes, compared with other GAD65Ab-positive phenotypes [13, 17-19]. Recently we described two conformational epitopes that are located in the middle region of GAD65 and were specifically recognised by GAD65Ab from type 1 diabetic patients [17]. However, very little is known about the development of GAD65Abs and their epitope specificity in the pre-diabetic period. The characterisation of monoclonal GAD65Ab from patients with type 1 diabetes demonstrated that these antibodies had undergone somatic mutations, indicating affinity maturation, during the progression to type 1 diabetes $[20,21]$. This gives rise to the question of whether disease-associated GAD65 Ab specificities are the product of antibody maturation, or an inherent characteristic of GAD65 Ab present in pre-diabetic individuals. In this longitudinal study, we compared GAD65Ab epitopes in children who are at high risk of developing diabetes with those present in children who are at low risk of developing the disease.

\section{Subjects and methods}

Human sera Serum samples were obtained from participants in the Karlsburg Type 1 Diabetes Risk Study. In this study, 9,419 randomly selected, healthy schoolchildren (617 years old) underwent screening for autoantibodies related to type 1 diabetes, and autoantibody-positive children were followed longitudinally as previously described [22, 23]. A total of 387 serum samples from 86 GAD65Abpositive children identified in this study were analysed for binding to human GAD65 and rat GAD67. Twenty-six individuals were excluded because of antibody reactivity with GAD67 ( $n=13)$, low GAD65Ab titre of the longitu-
Table 1 Study-related parameters of the analysed individuals

\begin{tabular}{lll}
\hline & High risk $(n=22)$ & Low risk $(n=38)$ \\
\hline $\begin{array}{l}\text { GAD65Ab titre (index) } \\
\text { Initial sample (range) }\end{array}$ & $0.7(0.1-1.9)$ & $0.3(0.1-1.5)$ \\
Final sample (range) & $0.8(0.22-1.8)$ & $0.4(0.1-2.0)$ \\
Median no. samples & $5(2-9)$ & $3(2-7)$ \\
per individual (min-max) & & \\
Follow-up period in & $48(2-55)$ & $42(11-55)$ \\
months (range) & & \\
Age in years (range) & $12(7-16)$ & $11(6-15)$ \\
Sex (female/male) & $11 / 11$ & $21 / 17$ \\
HLA-DQ risk genotype frequency $(n)$ & \\
$02 / 02$ & 2 & 3 \\
$02 / 0501$ & 2 & 2 \\
$02 / 0302$ & 4 & 5 \\
$0302 / 0304$ & 1 & 0 \\
$0302 / 0402$ & 1 & 0 \\
$0302 / 0501$ & 2 & 0 \\
$0302 / 0302$ & 2 & 1 \\
$0302 / 0502$ & 0 & 1 \\
& &
\end{tabular}

dinal samples $(n=10)$, or limited follow-up data, with the number of samples below $2(n=3)$ (Fig. 1). The removal of GAD65/67 cross-reactive sera was necessary as the recombinant Fabs ( $\mathrm{rFab})$ used in this study are GAD65-specific and do not bind to GAD67 [21, 24]. The GAD65Ab epitopes of the remaining 301 serum samples from $60 \mathrm{GAD} 65 \mathrm{Ab}$ positive children were analysed (Table 1). A median of four samples $(n=2-9)$ per individual was analysed. Children whose sera tested positive for more than one diabetes-related autoantibody in the initial screen (GAD65Ab, IA-2Ab, or insulin autoantibodies) were classified as high-risk individuals $(n=22)$ (Fig. 1). Of these, nine developed type 1 diabetes during the follow-up period. The last of these children developed diabetes after a follow-up of 55 months. The remaining children $(n=38)$ were positive for GAD65 $\mathrm{Ab}$ alone and therefore classified as low-risk individuals. None of these children developed type 1 diabetes in the followup or developed reactivity to additional autoantibodies. The study protocol was authorised by the Ministry of Culture and Education of Mecklenburg-Vorpommern and approved by the ethics committee of the Ernst-Moritz-Arndt-Univer-
Fig. 1 Flow-chart illustrating the different study groups used in this study

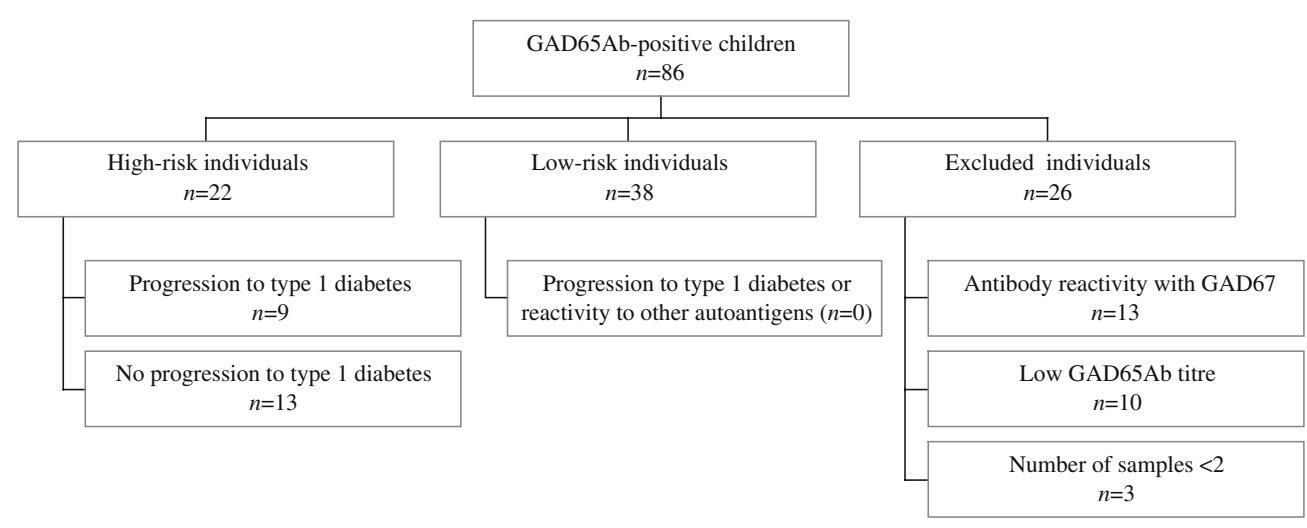


sity (Greifswald, Germany). Informed consent was obtained from the parents or legal guardians, and the investigations were carried out in accordance with the principles of the Declaration of Helsinki, as revised in 2000.

Expression and purification of the antibody $r F a b s$ The four rFabs used in this study have been described in detail [17]. Briefly, rFab DPA, DPD and DPC were isolated from a patient with type 1 diabetes [21]. These rFabs recognise epitopes at amino acid residues 483-585 (C-terminus), 96-173 (N-terminus), and 195-412 (middle) respectively [25]. b96.11 was isolated from a patient with autoimmune polyendocrine syndrome type 1 and recognises an epitope at amino acid residues 308-365 (middle) [24, 25]. rFabs were expressed in bacteria as previously described [17]. Briefly, E. coli 25F2 cells containing the recombinant plasmids were grown for $16 \mathrm{~h}$ at $30^{\circ} \mathrm{C}$ in complete MOPS medium [26]. Cells were then subcultured and grown in the absence of phosphate at $30^{\circ} \mathrm{C}$ for $4 \mathrm{~h}$. The rFabs were isolated from the bacteria as described previously [27] and purified by two subsequent affinity chromatography steps on Ni-NTA Agarose (Qiagen, Valencia, CA, USA) and protein G sepharose (PGS) (Zymed Laboratories, Carlton Court, CA, USA). Fractions were examined by immunoblot for the presence of $\mathrm{rFabs}$ and by radioligand binding test for GAD65 binding. Active fractions were pooled and the protein concentration was determined. The yield of functional purified $\mathrm{rFab}$ was about $0.5-1 \mathrm{mg} / \mathrm{l}$ bacterial culture.

Competition studies of rFab with monoclonal antibodies and serum Recombinant $\left[{ }^{35} \mathrm{~S}\right]$-labelled human GAD65 and rat GAD67 were produced in an in vitro coupled transcription/translation system with SP6 RNA polymerase and nuclease-treated rabbit reticulocyte lysate (Promega, Madison, WI, USA), as described previously $[28,29]$. The in vitro translated $\left[{ }^{35} \mathrm{~S}\right]$-antigen was stored at $-70^{\circ} \mathrm{C}$ and used within 2 weeks. Binding of $\mathrm{rFab}$ to radiolabelled antigen was determined as described previously, using PGS as the precipitating agent [17].

The capacity of the rFab to inhibit GAD65 binding by human serum GAD65 Ab was tested in a competitive radioligand binding test using protein A sepharose (Zymed Laboratories) to separate antibody-bound from free antigen. The rFabs were added at the optimal concentration (0.7-1 $\mu \mathrm{g} / \mathrm{ml})$ as determined in competition assays using the intact monoclonal antibody as a competitor. The background competition for each $\mathrm{rFab}$ was established in competition experiments with normal control sera. The background was subtracted prior to calculation of percentage inhibition. The cutoff for specific competition was set at greater than $15 \%$ by using as a negative control rFab NQ22/61.1 (a kind gift from Dr J. Foote, Fred Hutchinson Research Center, Seattle), specific to an irrelevant target, phenyl oxazolone, at $5 \mu \mathrm{g} / \mathrm{ml}$.

$H L A-D Q B 1$ typing HLA-DQB1 genotyping was performed by non-radioactive oligonucleotide hybridisation of enzymatically amplified DNA, as previously described [22]. Because DQB1 0201 and 0202 differ by a dimorphism at codon 135, located in the third exon, and are thus not differentiated by regular DQB1 genotyping, DQ2-positive individuals are given as DQB1 02. HLA-DQB1 risk genotypes were classified as follows (number of subjects with a particular genotype shown within parenthesis): 02/02 (5), 02/0302 (9), 02/0501 (4), 0302/0304 (1), 0302/0402 (1), 0302/0501 (2), 0302/0302 (3), 0302/0502 (1). HLA DQB1 genotypes with no or with a negative association with type 1 diabetes were: 0301/0301 (3), 0301/0402 (1), 0301/0302 (4), 0301/0602 (1), 0301/0501 (1),02/0301 (4), 02/0602 (3), 02/0402 (1), 02/0603 (1), 0501/0604 (1), 0304/02 (1), 0602/0602 (1), 0602/0503 (1), 0602/0303 (1), 0302/0603 (3), 0502/0201 (1), 0402/0501 (1), 0402/0604 (1),0601/ 0301 (1), 02/0604 (1), 0502/0602 (1), 0604/0503 (1).

Statistical analyses Binding of GAD65Ab to GAD65 in the presence of rFab was expressed as follows:

$$
\begin{aligned}
& \text { (cpm of } \left.\left[{ }^{35} S\right]-G A D 65 \text { bound in the presence of } r F a b\right) \\
& \quad /\left(\text { cpm of }\left[{ }^{35} S\right]-G A D 65 \text { bound in the absence of } r F a b\right) \\
& \times 100
\end{aligned}
$$

The GAD65Ab index was defined as follows: (cpm of tested sample-average cpm of 2 negative standards)/(cpm of positive standard-average cpm of 2 negative standards). The Juvenile Diabetes Foundation islet cell antibody standard [30], which is also GAD65Ab-positive, was used as the GAD65Ab-positive standard. A randomly selected control serum from a healthy volunteer was used as the negative standard.

All samples were analysed in triplicate determinations and the average intra-assay coefficient of variation was $4 \%$ (range $0.6-9 \%$ ). Positive and negative controls were included on each plate to correct for interassay variations. The upper level of normal (0.047) was established by analysing sera obtained from non-diabetic subjects $(n=50)$. In the third DASP Workshop our assay showed $84 \%$ sensitivity and $91 \%$ specificity for GAD65Ab. Differences in competition between different serum groups were tested for significance with the non-parametric Mann-Whitney $U$ test. Differences in competition within serum groups were tested using the Wilcoxon matched pair test. Linear regression curves were compared using the Prism statistical analysis tool (GraphPad Software, San Diego, CA, USA). A $p$ value of less than 0.05 was considered significant.

\section{Results}

GAD65Ab titre and epitopes recognised in healthy schoolchildren We first established the GAD65Ab titre (Table 1) and epitope specificity of all individuals at the initial sample. When comparing the level of competition achieved with individual rFabs in the first sample taken from children in both groups, no significant differences were observed (Fig. 2). No correlation was observed between the GAD65Ab titre and the level of competition achieved with any of the rFabs (data not shown). This is in accordance with our 
Fig. 2 Binding to GAD65 in the presence of rFab DPA (a), b96.11 (b), DPC (c), and DPD (d). Binding of initial samples from high-risk individuals (triangles), individuals that later progressed to type 1 diabetes (circles) and low-risk individuals (diamonds) is given in percent. Median binding is indicated by a horizontal line a
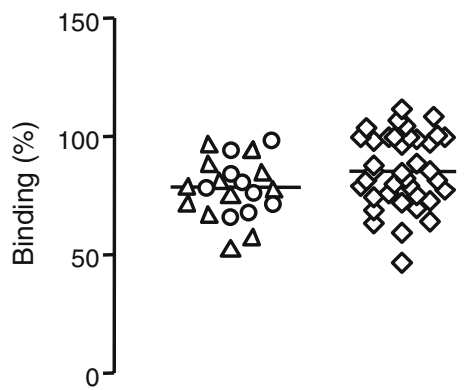

C

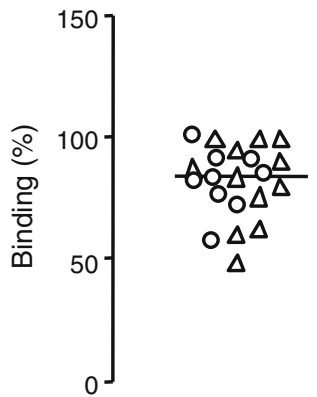

b

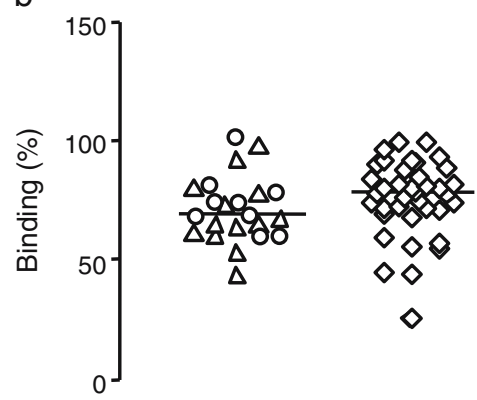

d
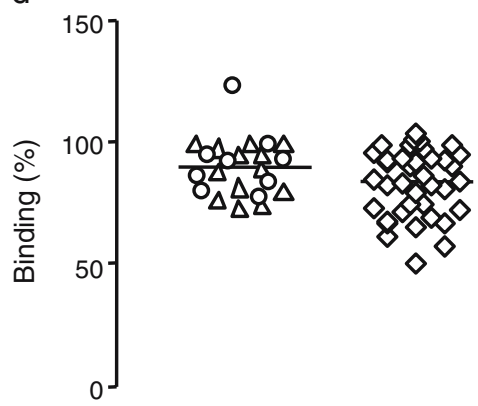

previous findings [17]. Finally, we evaluated whether there were any changes in the GAD65Ab titre over time. Because the last individual developed diabetes at 55 months of follow-up, we limited the period of analysis for all individuals accordingly. During this time period, no significant changes of GAD65Ab titre were observed for any of the groups (Table 1).

GAD65Ab epitope pattern in the initial sample While the GAD65Ab in both groups recognised similar epitopes, we found significant differences in particular combinations of epitope specificities present in individual patients when comparing the two groups. At the initial sampling, most of the sera in both groups had GAD65Abs that were competed with by a median of two rFabs. Nearly all samples (93$100 \%$ ) recognised epitopes located in the middle of the molecule, defined as competition by DPC and/or b96.11 (Fig. 3a, b). While the most frequent epitope combination for high-risk individuals in the initial sample included the C-terminus (competed with by rFab DPA) and the middle region (competed with by rFab b96.11; 70\% of the samples, shaded regions in Fig. 2a), only $37 \%$ of the low-risk individuals had GAD65Ab that were reactive with this epitope combination $(p<0.0001)$.

GAD65Ab epitope pattern in the last sample The differences in epitope combinations observed in the initial sample were even more pronounced upon analysis of the last sample. Both the number of epitopes and the epitope pattern recognised had changed for the high-risk individuals (Fig. 3c). While a median of two rFabs still competed with GAD $65 \mathrm{Ab}$ present in the sera of the low-risk individuals, a median of four rFabs competed with GAD65Ab present in the sera of high-risk individuals. Among the high-risk individuals, the percentage of sera in which all four rFabs competed with GAD65Abs increased from 13 to $60 \%(p<$ $0.0001)$ between the first and last sample. The remainder

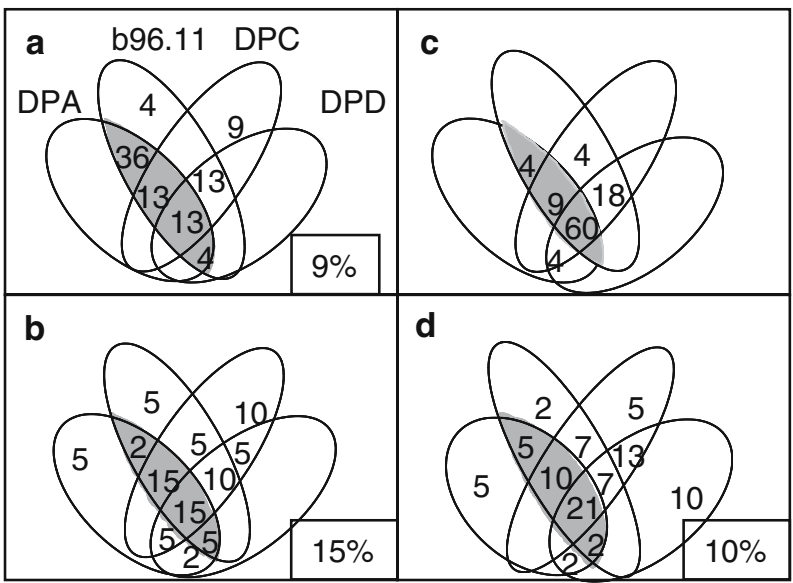

Fig. 3 Venn diagrams for sera from high-risk (a) and low-risk individuals (b) at the initial sample time and for sera from high-risk (c) and low-risk (d) individuals at the last sample time. Sera were analysed for competition with the indicated $\mathrm{rFab}$ to determine their combination of GAD65Ab specificities. The numbers in the diagrams depict the percentage of serum samples in each group that competed with the respective rFabs. Absolute numbers represented by the above percentages are: $(\mathbf{a}, \mathbf{c}): 60 \%(n=13), 36 \%(n=8), 18 \%(n=4)$, $13 \%(n=3), 9 \%(n=2)$, and $4 \%(n=1) ;(\mathbf{b}, \mathbf{d}): 21 \%(n=8), 15 \%(n=6)$, $13 \%(n=5), 10 \%(n=4), 7 \%(n=3), 5 \%(n=2), 2 \%(n=1)$. The shaded areas highlight the percentage of serum samples that competed with a combination of $\mathrm{rFab}$ b96.11 (middle-) and DPA (C-terminusspecific). The percentage of serum samples that were not competed with by any of the four rFabs are shown in a box at the lower right corner of each panel 
competed with different combinations of two or three rFabs in the last sample. The epitope pattern in the low-risk individuals remained unchanged between the initial and the last serum samples, with only $21 \%$ of the last samples having GAD65Abs competed with by all four rFabs, and $32 \%$ of the samples still competed with by one $\mathrm{rFab}$ or fewer.

High-risk individuals show significant epitope changes over time To compare the change of epitope recognition over time between different subject groups, we investigated the degree of competition conferred by each $\mathrm{rFab}$ by linear regression analysis (data not shown). For the low-risk individuals, we did not observe any significant change in degree of epitope binding using any of the four rFabs. On the other hand, significant increases in degree of epitope binding over time for epitopes represented by $\mathrm{rFab}$ b96.11, DPC and DPD were noted in the high-risk individuals ( $p$ values: $<0.0001,0.0035$ and $<0.0001$ respectively).

GAD65Ab specificities in the last sample from high-risk individuals differ from those found in low-risk individuals The change in epitope binding over time resulted in significant differences in competition levels. In the high-risk group, GAD65Abs with epitope specificities competed with by $\mathrm{rFab}$ b96.11, DPC and DPD were found more frequently in the last sample than in the initial sample, as assessed by degree of competition for each $\mathrm{rFab}(p$ value: $0.002,0.002$ and 0.001 respectively) (data not shown). However, no significant difference in epitope specificities between the first and the last sample of the low-risk individuals was observed for any of the rFabs.

Finally, when comparing the degree of epitope binding in the last sample, we observed a significant difference between both groups (Fig. 4). GAD65Abs in the sera of highrisk individuals were significantly better competed with by $\mathrm{rFab}$ b96.11 than those in sera of low-risk individuals

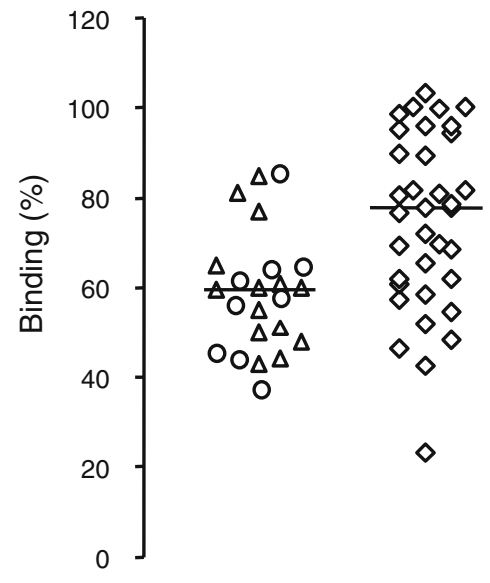

Fig. 4 Binding, in final serum samples, to GAD65 in the presence of $\mathrm{rFab}$ b96.11. Sera from high-risk individuals (left, triangles and circles) and low-risk individuals (right, diamonds) were analysed. Individuals that progressed to type 1 diabetes during follow-up are represented as white circles. Median binding is indicated by a horizontal line
( $p=0.001)$. No differences in the degree of epitope binding between groups were observed for the other three rFabs, and no differences were observed when comparing individuals in the high-risk group who progressed to diabetes with high-risk individuals who did not progress to diabetes.

Relationship between GAD65Ab specificities and risk HLA markers Possible associations between the HLA-DQB1 genotype and GAD65Ab specificity were analysed (Table 2). No association between individuals with the risk-conferring HLA genotype and GAD65Ab specific to the DPAdefined epitope was found. However, significantly more individuals with the risk-conferring HLA genotype showed an increase in binding to the b96.11-defined epitope over time as compared with individuals with HLA genotypes that were not or were or negatively associated with risk of type 1 diabetes ( $p=0.0004)$. The same association was found between individuals with the risk-conferring HLA genotype and the combination of GAD65Ab specific to the DPA- and b96.11-defined epitopes ( $p=0.0018)$.

To better understand how the dynamic changes in epitope binding compare with conventional measures of risk, we analysed the sensitivity and specificity for risk defined by multiple islet autoantibodies. We found that in $72 \%$ $(16 / 22)$ of the high-risk individuals GAD65Ab showed an increase in binding to the b96.11-defined epitope, while this was observed only for $10 \%(4 / 38)$ of the low-risk individuals. This results in a positive predictive value of $80 \%$. In our study population the HLA-genotype analysis comes to a positive predictive value of $54 \%$ (Table 1 ).

Epitope specificities change gradually with no correlation with time before onset When correlating changes in epitope specificities with time before clinical onset of the disease in individual patients, no clear relationship was observed (Fig. 5). One individual had a dramatic increase in binding to all four epitopes within the last 2 months before diag-

Table 2 Association between HLA genotype and GAD65Ab specificity

\begin{tabular}{lll}
\hline & $\begin{array}{l}\text { Risk HLA } \\
\text { genotype } \\
(n=26)\end{array}$ & $\begin{array}{l}\text { Not associated or } \\
\text { negatively associated } \\
\text { with type 1 diabetes } \\
(n=34)\end{array}$ \\
\hline $\begin{array}{l}\text { Sera specific to } \\
\text { DPA-defined epitope } \\
\text { (first sample) }\end{array}$ & 18 & 17 \\
$\begin{array}{l}\text { Sera with increasing } \\
\text { GAD65Ab specificity } \\
\text { to b96.11-defined } \\
\text { epitope (over time) }\end{array}$ & 14 & 6 \\
$\begin{array}{l}\text { Sera specific to b96.11- } \\
\text { and DPA-defined epitopes } \\
\text { (first sample) }\end{array}$ & 17 & 12 \\
$\begin{array}{l}\text { Median GAD65Ab } \\
\text { titre (range) }\end{array}$ & $0.37(0.14-1.8)$ & $0.27(0.1-1.6)$ \\
\hline
\end{tabular}


Fig. 5 a-v Epitope specificities of the high-risk individuals, shown as a function of time. Panels show binding to GAD65 in the presence of $\mathrm{rFab}$ DPA (white squares), b96.11 (black squares), DPC (white circles), and DPD (black circles). Nine of the individuals progressed to type 1 diabetes $(\mathbf{a}-\mathbf{i})$, the time of clinical diagnosis being indicated by the arrow
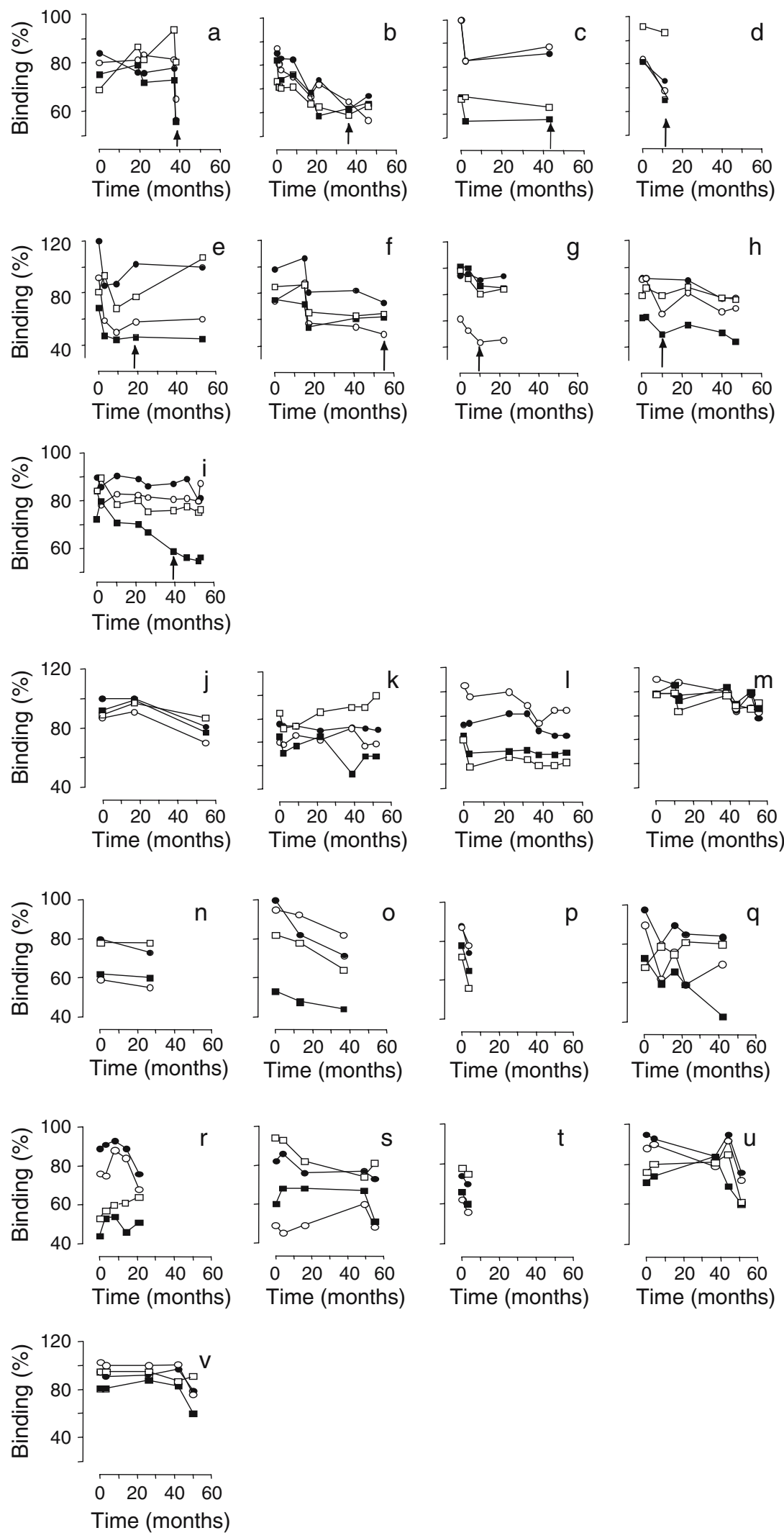

nosis (Fig. 5a). Most of the other individuals exhibited a more gradual change in their epitope specificities over time. For five individuals, samples were available after onset of the disease (Fig. 5b, e, g-i). No further significant change in epitope specificities was observed after onset of disease, with the exception of one child, in whom a decrease in binding to the DPA-recognised C-terminal epitope was observed (Fig. 5e). 


\section{Discussion}

Our previous analysis of disease-associated GAD65Ab epitopes in type 1 diabetes revealed two conformational epitopes that are located in the middle of the molecule and were specifically recognised by sera of type 1 diabetes patients [17]. In the present study, we addressed the question of whether this epitope pattern develops as an individual progresses to type 1 diabetes, or whether it is an inherent feature of the pre-diabetic GAD65 Ab response. We investigated GAD65 Ab epitope profiles in sera obtained from a longitudinal study of GAD65Ab-positive school children, some of whom were at higher risk of progressing to type 1 diabetes, as defined by multiple islet autoantibody positivity.

In high-risk individuals, of whom more than $40 \%$ developed diabetes during the follow-up period, we observed that: (1) sera recognise a specific combination of GAD65 Ab epitopes directed towards a middle epitope and a C-terminal epitope (recognised by $\mathrm{rFab}$ b96.11 and DPA respectively); (2) high risk of progression to disease is associated with the emergence of antibodies specific for conformational epitopes at the $\mathrm{N}$-terminus and the middle region; and (3) the degree of binding to already established $\mathrm{Ab}$ epitopes located in the middle and the $\mathrm{N}$-terminus increases in high-risk individuals, but not in low-risk individuals. These $\mathrm{Ab}$ binding characteristics may reflect epitope spreading and antibody maturation. No significant differences in the epitope binding pattern or in the longitudinal changes in epitope binding were observed between progressors and non-progressors in the high-risk group. The remarkably stable epitope pattern in the low-risk individuals is in agreement with a previous report showing that GAD65Abpositive individuals who did not progress to type 1 diabetes demonstrated a similarly stable epitope reactivity [31].

Intramolecular Ab epitope spreading is a phenomenon in which new epitopes within the same molecule are recognised over time (reviewed in [32, 33]). While this has been well demonstrated in various experimentally induced or spontaneous animal models of autoimmunity, the evidence for epitope spreading in human autoimmune diseases is limited (reviewed in [34]). Possible intramolecular Ab epitope spreading has been reported in patients with systemic lupus erythematosus [35]. Intramolecular Ab epitope spreading associated with progression to disease was recently reported for endemic pemphigus foliaceus (fogo selvagem) [36]. Because of the difficulty in identifying sufficient numbers of individuals that have been longitudinally followed until onset of type 1 diabetes, GAD65Ab reactivity during the pre-diabetic period has remained poorly defined. In the few studies addressing this issue, no specific changes in GAD65 Ab reactivities were observed as the individuals progressed to disease onset $[31,37,38]$. However, in these previous reports, GAD65/67 fusion proteins were used to analyse GAD65Ab epitope binding. Comparing GAD65Ab epitope analysis using GAD65/67 fusion proteins and GAD65-specific rFabs, we observed that the conformational epitopes located in the middle of GAD65 are very sensitive to conformational changes and may not be well preserved in fusion proteins [39]. The disease-specific GAD65Abs that recognise these conformational epitopes may therefore not bind to the fusion protein; thus, changes in GAD65Ab specificity to these middle epitopes would not be detected.

While binding to the C-terminal epitope recognised by DPA appears to be unaffected, the recognition of both middle (DPC and b96.11) and N-terminal (DPD) autoantibody epitopes progressively increases during progression to diabetes. The finding of epitope spreading towards N-terminal epitopes confirms results from an earlier study of pre-diabetic children of parents with type 1 diabetes [31]. Early reactivity to the middle and C-terminal epitopes of GAD65 has previously been described in offspring of parents with type 1 diabetes [14, 18, 37, 40]. Since the inclusion criterion for our study was GAD65Ab positivity, we do not have data on the transition from GAD65Ab negativity to GAD65Ab positivity. Therefore, the question of whether GAD65Ab reactivity to the C-terminal and middle region of the molecule arise together or successively cannot be addressed in this study.

To our knowledge, this is the first time that GAD65Ab epitope changes specifically associated with the risk of developing diabetes have been described. These changes occurred at different times before the onset of disease in those individuals who developed the disease. While in most cases, the epitope specificities changed gradually, in some cases rapid changes in epitope recognition occurred months or years before onset. It is notable that the epitope specificities remained relatively stable after the clinical onset of disease, which is in agreement with previous findings $[41,42]$.

We conclude that the GAD65Ab response in high-risk individuals is dynamic. The cause of these dynamic changes in GAD65Ab binding remains unclear. Two possible contributors to this phenomenon are antigen presentation specific to genetically predisposed individuals and environmental factors. The association between risk-conferring HLA types and GAD65Ab epitope specificity may explain previous studies demonstrating an earlier age at onset in children with high-risk HLA [43-45]. The age-dependent risk between HLA and type 1 diabetes is not understood but may be marked by an HLA-dependent development of epitopespecific GAD65Ab. The dynamic changes in binding to the b96.11-defined epitope may contribute to our ability to predict risk, apart from the standard risk assessment based on HLA genotype.

Acknowledgements This study was supported by the Juvenile Diabetes Foundation International (1-2001-873) and the National Institute of Health (grants DK26190 and DK53004 to Åke Lernmark in whose laboratory the work for this study was conducted), and by a Career Development Award from the American Diabetes Association for C. S. Hampe M. Schlosser was supported by the Deutsche Diabetes-Stiffung and the Else Kröner-Fresenius Stiftung. Support for the immunogenetic investigations was obtained from the BMFTfunded Center for Interdisciplinary Clinical Research at the FriedrichAlexander-Universität Erlangen-Nürnberg (IZKF Erlangen), Germany. We gratefully acknowledge Dr Åke Lernmark for helpful advice and critical reading of the manuscript. 


\section{References}

1. Riley WJ, Maclaren NK, Krischer J et al (1990) A prospective study of the development of diabetes in relatives of patients with insulin-dependent diabetes. N Engl J Med 323:1167-1172

2. Verge CF, Gianani R, Yu L et al (1995) Late progression to diabetes and evidence for chronic beta-cell autoimmunity in identical twins of patients with type I diabetes. Diabetes 44: $1176-1179$

3. Baekkeskov S, Landin-Olsson M, Kristensen JK et al (1987) Antibodies to a $64,000 \mathrm{Mr}$ human islet cell antigen precede the clinical onset of insulin-dependent diabetes. J Clin Invest 79: 926-934

4. Atkinson MA, Maclaren NK, Scharp DW, Lacy PE, Riley WJ (1990) 64,000 $\mathrm{M}_{\mathrm{r}}$ autoantibodies as predictors of insulindependent diabetes. Lancet 335:1357-1360

5. Bingley PJ, Christie MR, Bonifacio E et al (1994) Combined analysis of autoantibodies improves prediction of IDDM in islet cell antibody-positive relatives. Diabetes 43:1304-1310

6. Schatz D, Krischer J, Horne G et al (1994) Islet cell antibodies predict insulin-dependent diabetes in United States school-age children as powerfully as in unaffected relatives. J Clin Invest 93:2403-2407

7. Verge CF, Gianani R, Kawasaki E et al (1996) Prediction of type I diabetes in first-degree relatives using a combination of insulin, GAD, and ICA512bdc/IA-2 autoantibodies. Diabetes 45:926933

8. Kulmala P, Savola K, Petersen JS et al (1998) Prediction of insulin-dependent diabetes mellitus in siblings of children with diabetes - a population-based study. J Clin Invest 101:327-336

9. LaGasse JM, Brantley MS, Leech NJ et al (2002) Successful prospective prediction of type 1 diabetes in schoolchildren through multiple defined autoantibodies: an 8-year follow-up of the Washington State Diabetes Prediction Study. Diabetes Care 25:505-511

10. Jaume JC, Parry SL, Madec AM, Sonderstrup G, Baekkeskov S (2002) Suppressive effect of glutamic acid decarboxylase 65specific autoimmune B lymphocytes on processing of $\mathrm{T}$ cell determinants located within the antibody epitope. J Immunol 169:665-672

11. Banga JP, Moore JK, Duhindan N et al (2004) Modulation of antigen presentation by autoreactive $\mathrm{B}$ cell clones specific for GAD65 from a type I diabetic patient. Clin Exp Immunol 135: $74-84$

12. Reijonen H, Daniels TL, Lernmark A, Nepom GT (2000) GAD65-specific autoantibodies enhance the presentation of an immunodominant T-cell epitope from GAD65. Diabetes 49: $1621-1626$

13. Hampe CS, Hammerle LP, Bekris L et al (2000) Recognition of glutamic acid decarboxylase (GAD) by autoantibodies from different GAD antibody-positive phenotypes. J Clin Endocrinol Metab 85:4671-4679

14. Sohnlein P, Muller M, Syren K et al (2000) Epitope spreading and a varying but not disease-specific GAD65 antibody response in Type I diabetes. The Childhood Diabetes in Finland Study Group. Diabetologia 43:210-217

15. Tree TI, Morgenthaler NG, Duhindan N et al (2000) Two amino acids in glutamic acid decarboxylase act in concert for maintenance of conformational determinants recognised by Type I diabetic autoantibodies. Diabetologia 43:881-889

16. Primo ME, Anton EA, Villanueva AL, Poskus R, Ermacora MR (2003) Engineered variants of human glutamic acid decarboxylase (GAD) and autoantibody epitope recognition. Clin Immunol 108:38-45

17. Padoa CJ, Banga JP, Madec AM et al (2003) Recombinant Fabs of human monoclonal antibodies specific to the middle epitope of GAD65 inhibit type 1 diabetes-specific GAD65Abs. Diabetes 52:2689-2695

18. Falorni A, Ackefors M, Carlberg C et al (1996) Diagnostic sensitivity of immunodominant epitopes of glutamic acid decarboxylase (GAD65) autoantibodies epitopes in childhood IDDM. Diabetologia 39:1091-1098
19. Powers AC, Bavik K, Tremble J, Daw K, Scherbaum WA, Banga JP (1999) Comparative analysis of epitope recognition of glutamic acid decarboxylase (GAD) by autoantibodies from different autoimmune disorders. Clin Exp Immunol 118:349-356

20. Richter W, Jury KM, Loeffler D, Manfras BJ, Eiermann TH, Boehm BO (1995) Immunoglobulin variable gene analysis of human autoantibodies reveals antigen-driven immune response to glutamate decarboxylase in type 1 diabetes mellitus. Eur J Immunol 25:1703-1712

21. Madec AM, Rousset F, Ho S et al (1996) Four IgG anti-islet human monoclonal antibodies isolated from a type 1 diabetes patient recognise distinct epitopes of glutamic acid decarboxylase 65 and are somatically mutated. J Immunol 156:3541-3549

22. Schlosser M, Strebelow M, Wassmuth R et al (2002) The Karlsburg type 1 diabetes risk study of a normal schoolchild population: association of beta-cell autoantibodies and human leukocyte antigen-DQB1 alleles in antibody-positive individuals. J Clin Endocrinol Metab 87:2254-2261

23. Strebelow M, Schlosser M, Ziegler B, Rjasanowski I, Ziegler M (1999) Karlsburg Type I diabetes risk study of a general population: frequencies and interactions of the four major Type I diabetes-associated autoantibodies studied in 9,419 schoolchildren. Diabetologia 42:661-670

24. Tremble J, Morgenthaler NG, Vlug A et al (1997) Human B cells secreting immunoglobulin $\mathrm{G}$ to glutamic acid decarboxylase65 from a non-diabetic patient with multiple autoantibodies and Graves' disease: a comparison with those present in type 1 diabetes. J Clin Endocrinol Metab 82:2664-2670

25. Schwartz HL, Chandonia JM, Kash SF et al (1999) Highresolution autoreactive epitope mapping and structural modeling of the $65 \mathrm{kDa}$ form of human glutamic acid decarboxylase. J Mol Biol 287:983-999

26. Neidhardt FC, Bloch PL, Smith DF (1974) Culture medium for enterobacteria. J Bacteriol 119:736-747

27. Carter P, Kelley RF, Rodrigues ML et al (1992) High level Escherichia coli expression and production of a bivalent humanized antibody fragment. Biotechnology (NY) 10:163-167

28. Grubin CE, Daniels T, Toivola B et al (1994) A novel radioligand binding assay to determine diagnostic accuracy of isoform-specific glutamic acid decarboxylase antibodies in childhood IDDM. Diabetologia 37:344-350

29. Falorni A, Örtqvist E, Persson B, Lernmark A (1995) Radioimmunoassays for glutamic acid decarboxylase (GAD65) and GAD65 autoantibodies using ${ }^{35} \mathrm{~S}$ or ${ }^{3} \mathrm{H}$ recombinant human ligands. J Immunol Methods 186:89-99

30. Mire-Sluis AR, Gaines Das R, Lernmark A (2000) The World Health Organization International Collaborative Study for islet cell antibodies. Diabetologia 43:1282-1292

31. Bonifacio E, Lampasona V, Bernasoni L, Ziegler AG (2000) Maturation of the humoral autoimmune response to epitopes of GAD in preclinical childhood Type 1 diabetes. Diabetes 49:202208

32. Vanderlugt CJ, Miller SD (1996) Epitope spreading. Curr Opin Immunol 8:831-836

33. Mamula MJ (1998) Epitope spreading: the role of self peptides and autoantigen processing by B lymphocytes. Immunol Rev 164:231-239

34. McCluskey J, Farris AD, Keech CL et al (1998) Determinant spreading: lessons from animal models and human disease. Immunol Rev 164:209-229

35. Arbuckle MR, Reichlin M, Harley JB, James JA (1999) Shared early autoantibody recognition events in the development of anti$\mathrm{Sm} \mathrm{B} / \mathrm{B}^{\prime}$ in human lupus. Scand J Immunol 50:447-455

36. Li N, Aoki V, Hans-Filho G, Rivitti EA, Diaz LA (2003) The role of intramolecular epitope spreading in the pathogenesis of endemic pemphigus foliaceus (fogo selvagem). J Exp Med 197: $1501-1510$

37. Hoppu S, Ronkainen MS, Kulmala P, Akerblom HK, Knip M (2004) GAD65 antibody isotypes and epitope recognition during the prediabetic process in siblings of children with type I diabetes. Clin Exp Immunol 136:120-128 
38. Achenbach P, Warncke K, Reiter J et al (2004) Stratification of type 1 diabetes risk on the basis of islet autoantibody characteristics. Diabetes 53:384-392

39. Binder K, Banga JP, Madec AM, Ortqvist E, Duo D, Hampe CS (2004) Epitope analysis of GAD65Ab using fusion proteins and rFab. J Immunol Methods 295:101-109

40. Richter W, Shi Y, Bækkeskov S (1993) Autoreactive epitopes defined by diabetes-associated human monoclonal antibodies are localized in the middle and C-terminal domains of the smaller form of glutamate decarboxylase. Proc Natl Acad Sci U S A 90:2832-2836

41. Hampe CS, Hammerle LP, Bekris L, Ortqvist E, Persson B, Lernmark A (2002) Stable GAD65 autoantibody epitope patterns in type 1 diabetes children five years after onset. J Autoimmun 18:49-53

42. Ronkainen MS, Savola K, Knip M (2004) Antibodies to GAD65 epitopes at diagnosis and over the first 10 years of clinical type 1 diabetes mellitus. Scand J Immunol 59:334-340
43. Graham J, Kockum I, Sanjeevi CB et al (1999) Negative association between type 1 diabetes and HLA DQB1*0602DQA $1 * 0102$ is attenuated with age at onset. Swedish Childhood Diabetes Study Group. Eur J Immunogenet 26:117-127

44. Van der Auwera BJ, Schuit FC, Weets I, Ivens A, van Autreve JE, Gorus FK (2002) Relative and absolute HLA-DQA1-DQB1 linked risk for developing type I diabetes before 40 years of age in the Belgian population: implications for future prevention studies. Hum Immunol 63:40-50

45. Pitkaniemi J, Hakulinen T, Nasanen J, Tuomilehto-Wolf E, Tuomilehto T (2004) Class I and II HLA genes are associated with susceptibility and age at onset in Finnish families with type 1 diabetes. Hum Hered 57:69-79 